
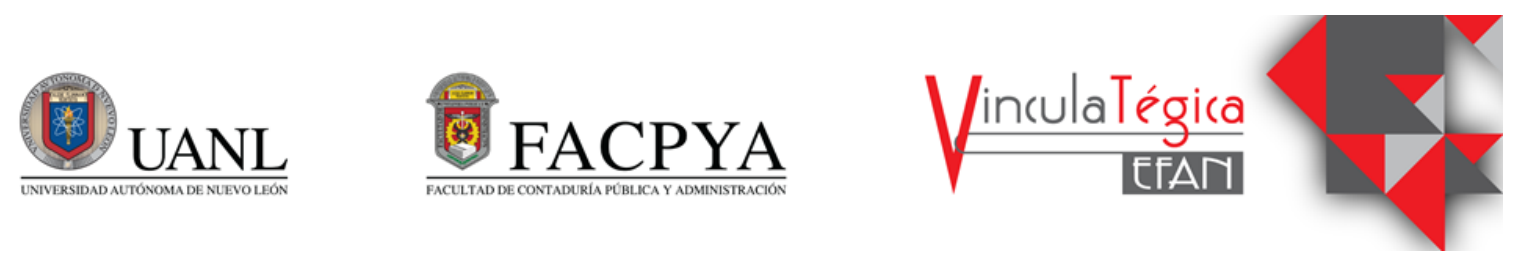

\title{
Estrategia de nodo de impulso a la economía social y solidaria para MiPyMES ante pandemia: artesanos de la región Pátzcuaro
}

\author{
Martin Tapia Salazar ${ }^{1}$, Laura Adame Rodríguez ${ }^{2}$ y Maricela Villanueva Pimentel ${ }^{3}$ \\ ${ }^{I}$ Tecnológico Nacional de México/Instituto Tecnológico Superior de Pátzcuaro, mtapia@itspa.edu.mx, Av. \\ Tecnológico No.1, Zurumutaro, CP.61615, Pátzcuaro Michoacán, Tel. 4341206860. \\ ${ }^{2}$ Tecnológico Nacional de México/Instituto Tecnológico Superior de Pátzcuaro, ladame@itspa.edu.mx, Av. \\ Tecnológico No.1, Zurumutaro, CP.61615, Pátzcuaro Michoacán, Tel. 4432272866. \\ ${ }^{3}$ Tecnológico Nacional de México/Instituto Tecnológico Superior de Pátzcuaro, mvillanueva@itspa.edu.mx, \\ Av. Tecnológico No.1, Zurumutaro, CP.61615, Pátzcuaro Michoacán, Tel. 434115955.
}

Información del artículo revisado por pares

Fecha de aceptación: junio-2021

Fecha de publicación en línea: diciembre-2021

DOI: https://doi.org/10.29105/vtga7.2-38

\section{Resumen}

Las micro, pequeñas y medianas empresas (MiPyMES) de la región de Pátzcuaro Michoacán han sido severamente afectadas durante la pandemia COVID-19, que ha traído consigo cambios en las estructuras: políticas, económicas y sociales, en donde, la modificación del modelo de negocios resulta ser una estrategia prioritaria. La presente investigación tiene como objeto de estudio una unión de artesanos de la región conformada por 19 negocios y como objetivo evidenciar los resultados de intervención de un NODESS como estrategia para las MiPyMES en una unión de artesanos de la región Pátzcuaro Michoacán, ante la pandemia COVID-19. Para lograr el objetivo se desarrolló una metodología basada en 4 etapas: a1) Talleres sobre economía social solidaria y funcionamiento de NODESS; 2) Diagnóstico; 3) Análisis estadístico de datos recolectados; y 4) Propuesta de mejora. En donde, se obtuvo como resultado una propuesta para mejorar la funcionalidad de los negocios.

\begin{abstract}
The micro, small and medium-sized enterprises (MiPyMES) of the Pátzcuaro Michoacán region have been severely affected during the COVID19 pandemic, which has brought about changes in the structures: political, economic and social, where, the modification of the model of business turns out to be a priority strategy. The present research aims to study a union of artisans from the region made up of 19 businesses and the objective of showing the results of the intervention of a NODESS as a strategy for MSMEs in a union of artisans from the Pátzcuaro Michoacán region, in the face of the COVID pandemic -19. To achieve the objective, a methodology based on 4 stages was developed: a1) Workshops on solidarity social economy and operation of NODESS; 2) Diagnosis; 3) Statistical analysis of collected data; and 4) Proposal for improvement. Where, as a result, a proposal was obtained to improve the functionality of the businesses.
\end{abstract}

Keywords: Covid-19, Social Economy, Solidarity Economy, Strategy, MSMEs. 
Palabras clave: Covid-19, Economía social, Economía Solidaria, Estrategia, Mipymes.

\section{INTRODUCCIÓN}

Las micro, pequeñas y medianas empresas (MIPyMES) de la región de Pátzcuaro Michoacán, han sido severamente afectadas durante la pandemia que se ha vivido en los últimos tiempos, muchos de estos negocios han visto reducidas en gran proporción sus utilidades y otros han desaparecido.

Acostumbradas a generar su mayor fuente de ingresos a través de las ventas realizadas a los visitantes nacionales e internacionales, actualmente y ante la disminución de turistas compradores, el empresario se ve obligado a buscar nuevas estrategias y alternativas de publicidad, comercialización, planeación y administración, realizando además alianzas estratégicas con los diferentes sectores de la sociedad, que le permitan afrontar estos nuevos retos.

Las Instituciones de educación superior juegan un papel importante en el impulso de la economía del sector empresarial y social de toda región. El Instituto Tecnológico Superior de Pátzcuaro (ITSPA), a través de un grupo de docentes investigadores, y tomando como base la autorización del INAES (Instituto Nacional de la Economía Social) para la conformación del Nodo de Impulso a la Economía Social y Solidaria (NODESS), y la Red Nacional de Nodos de Impulso a la Economía Social y Solidaria llevó a cabo una estrategia de intervención bajo este esquema, para una unión de artesanos conformada por 19 negocios de la región Pátzcuaro Michoacán, ante esta situación de pandemia COVID-19

$\mathrm{La}$ finalidad de la mencionada intervención, consistió en apoyar a la unión en la mejora y crecimiento de los negocios, incidiendo en el Objetivo 8 "trabajo decente y crecimiento económico", de los Objetivos de Desarrollo Sostenible (ODS), "Los Objetivos de Desarrollo Sostenible (ODS) adoptados por las Naciones Unidas en 2015 contienen la
JEL: A13, D41, D81, I10, I12.

agenda global más ambiciosa aprobada por la comunidad internacional para movilizar la acción colectiva en torno a objetivos comunes" (Gómez Gil, 2018, 107).

El presente trabajo muestra la metodología aplicada a los negocios que conforman la unión de artesanos, las actividades realizadas en conjunto, la implementación de estrategias para que los empresarios realizarán una planeación mejor organizada de sus actividades y aplicaran alternativas de venta con el uso de las Tic's para mejorar la rentabilidad de las MIPyMES, así mismo se muestran los resultados de la intervención realizada al grupo objeto de investigación.

\section{MARCO TEÓRICO}

Las condiciones del contexto global derivadas de la pandemia del COVID-19 han traído consigo cambios en las estructuras: políticas, económicas y sociales para los diferentes países. En donde, uno de los sectores que se ha visto mayormente golpeado es el empresarial, principalmente en su estratificación de MIPyMES por las características propiamente del mismo.

Esta situación, además, ha traído consigo la reformulación de la estrategia básica del modelo de negocios con el que se había venido operando, de tal manera que, se pueda garantizar la permanencia en un mercado con una alta especulación de riesgo. En un estudio realizado a MIPyMES del sector restaurantero de Tabasco, México demostró que la estrategia adoptada durante el primer trimestre de pandemia se centra en la innovación en procesos (De la Cruz \& May, 2021).

\subsection{Las MIPyMES ante el Covid-19}

Según Guardado, Martínez \& Tapia (2020), una de las repercusiones de esta pandemia para México hablando en términos empresariales, es el factor humano, ya que este mantiene implicaciones asociadas a la incorporación 
laboral posterior al periodo de aislamiento social, que van desde garantizar la salud física como mental de trabajadores.

Lo anterior, altera la operatividad interna de toda empresa, no obstante, es indispensable que este sector en el afán de garantizar las condiciones antes señaladas, se mantenga en una búsqueda de innovación con estrategias asociadas al mercado y la conservación de su posicionamiento.

Si bien la innovación no es un tema de estudio reciente para las organizaciones, en la actualidad existe una asociación directa con el uso de las Tecnologías de la Información y la Comunicación (Tic's) para su adopción (León \& Palma, 2018). El uso de herramientas de Tic's, en las MIPyMES ha ido incrementando significativamente, situación que se potencializa con las condiciones actuales de un mercado fluctuante frente a una pandemia.

En un estudio realizado a MIPyMES de Colombia sobre la incorporación del marketing digital en esta estratificación empresarial, se concluye que la incorporación de este tipo de herramientas como nuevo modelo operativo es indispensable por las condiciones actuales, sin embargo, se identifica la necesidad de intervenir para disminuir el paradigma que se tiene con respecto a la adopción de las mismas (Ochoa, Medina, Santamaria, Ramírez, Paramo y Ochoa, 2020).

De igual manera, en una investigación realizada a MIPyMES ecuatorianas en las que el confinamiento social tuvo un alto impacto, se demostró que el modelo de comercialización tradicional quedó fuera de la estrategia prioritaria para las empresas, siendo el comercio electrónico la base de restructuración de un nuevo modelo de negocio (Rodríguez, Ortiz, Quiroz y Parrales, 2020).

Con este modelo de negocio, además se pueden direccionar estratégicamente los objetivos de creación de nuevos productos o mercados; los canales de distribución y la disminución de costos en las actividades de las empresas (Bojórquez \& Valdez, 2017, citados en Anzules et.al., 2020)

$$
\text { Aunado a ello, Villegas-Garza }
$$

(2020), afirma que la migración de la empresa física a la modalidad virtual deberá estar acompañada de un cambio de la filosofía para propiciar una experiencia de compra online.

Como se hace notar en este apartado, ante esta contingencia sanitaria mundial, la transición del modelo de negocios implica adoptar nuevas estrategias empresariales y de apoyo con otros sectores, de tal manera que sirva como fortalecimiento y potenciación de sus recursos e infraestructura organizacional.

\subsection{Estrategia Nodo de Impulso a la Economía Social y Solidaria (NODESS)}

Las condiciones actuales a las que el sector empresarial se encuentra expuesto no han sido fáciles, el riesgo latente producto de una crisis económica da origen a nuevos modelos de organización, en donde, la prioridad es buscar permanecer en un mercado fluctuante, siendo la Economía Social una alternativa que ha resultado factible y de suma importancia en esta situación de pandemia COVID-19. Con la Economía Solidaria, las empresas se “(...) hacen más resistentes ante la crisis y se contribuye con una mayor capacidad para el mantenimiento del empleo" (Pérez González, 2011, 19).

Según Corrons (2016, citado en Medina y Flores, 2020), la economía social y solidaria tiene un gran potencial que integra a las personas, medio ambiente y desarrollo sostenible prioritariamente a los intereses meramente económicos.

Lo anterior, se atribuye entre otras cosas a que de acuerdo a los términos de referencia, "El NODESS se conforma por al menos tres actores representativos de los sectores social, académico y gubernamental, tales como:

- Un Organismo del Sector Social de la Economía (OSSE),

- Una Institución de educación superior y/o investigación, pública o privada,

- Una instancia del gobierno local (estatal o municipal)" (INAES, 2020).

Tal como se muestra en la figura que se presenta a continuación. 
Figura 1. Esquema de operación del NODESS.

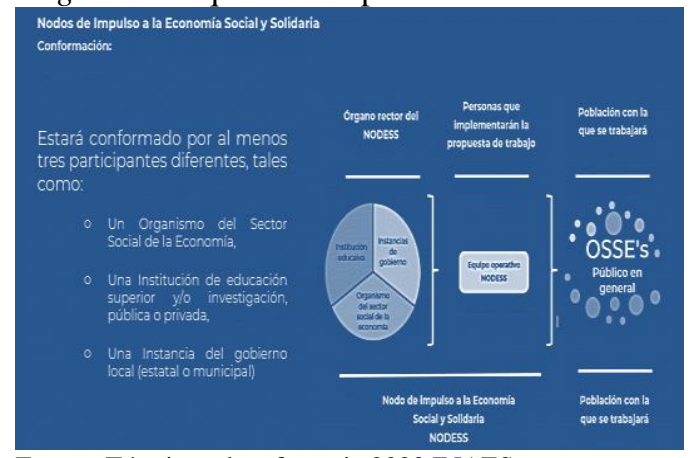

Fuente: Términos de referencia 2020 INAES.

Con ello, se enfatiza en crear las condiciones para generar un desarrollo económico que va más allá de un crecimiento económico centrado en aspectos específicamente de producción. En este sentido Pérez-González (2018), afirma que "El concepto de crecimiento económico plantea importantes diferencias respecto al de desarrollo sostenible. Este último abarca no sólo variables económicas, sino repercusiones personales, sociales, medioambientales y de calidad de vida, en general. Pero ambas perspectivas plantean en común el ámbito de aplicación: cuando se habla tanto de crecimiento económico como de desarrollo sostenible, siempre debe hacerse referencia al territorio como sustento económico y social".

\section{MÉTODO}

En la presente investigación se planteó como objetivo, evidenciar los resultados de intervención de un NODESS como estrategia para las MIPyMES en una unión de artesanos de la región Pátzcuaro Michoacán, ante la pandemia COVID-19.

La intervención se desarrolló encabezada por el Instituto Tecnológico Superior de Pátzcuaro (ITSPA), a través de un grupo de docentes investigadores, y tomando como base la autorización del INAES (Instituto Nacional de la Economía Social) para la conformación del Nodo de Impulso a la Economía Social y Solidaria (NODESS), y la Red Nacional de Nodos de Impulso a la Economía Social y Solidaria. Con el objeto de estudio de 19 negocios de la unión antes mencionada.

Para lograr la intervención, se aplicó una metodología basada en 4 etapas (como se muestra en la figura 2).

Figura 2. Metodología de intervención de NODESS.

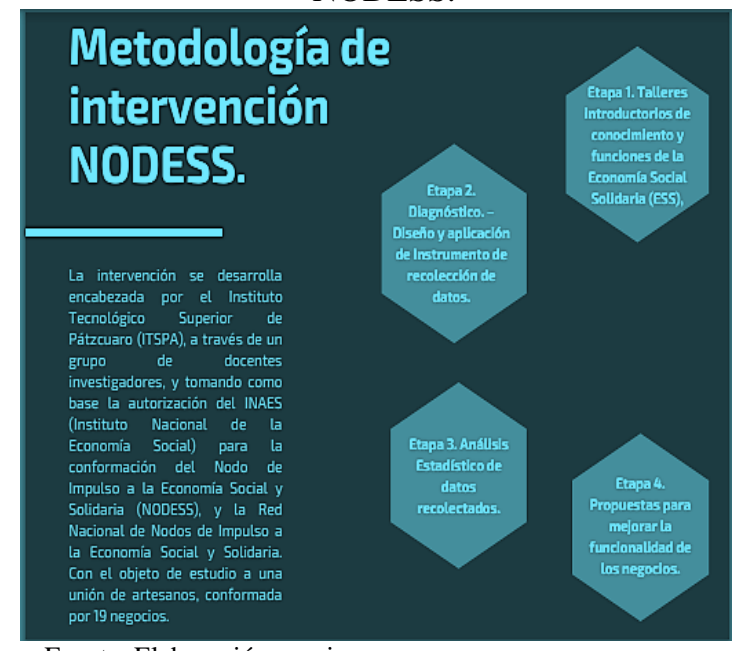

Fuente: Elaboración propia.

Etapa 1. Talleres introductorios de conocimiento y funciones de la Economía Social Solidaria (ESS); conformación y funcionalidad del NODESS Pátzcuaro

Michoacán. En esta etapa se realizaron diversas pláticas con dueños de los negocios, en las que se manejaron temas sobre: a) Sistema Nacional de Capacitación y Asistencia Técnica (SINCA), b) Conformación de una Red Nacional de Nodos de Impulso a la Economía Social y Solidaria (NODESS), coordinados por el INAES, c) Conformación y participantes del NODESS Pátzcuaro Michoacán, y d) objetivo, estrategias y órgano rector del NODESS Pátzcuaro.

Etapa 2. Diagnóstico sobre el estado actual de las empresas. En esta parte de la metodología, se realizaron actividades que permitieron diagnosticar la situación para cada uno de los negocios que conforman la unión de artesanos, en donde, se identificaron las necesidades y áreas de oportunidad para mejorar sus procesos, planeaciones y economía.

Para ello, se diseñó un instrumento de recolección de datos nombrado para este 
efecto como instrumento de medición de gestión empresarial (IMGE), tomando como base el instrumento de recolección de datos de Adame (2017). El IMGE está integrado por 49 ítems, dividido en cinco dimensiones y 12 indicadores. y antes de su aplicación a los empresarios fue evaluado para analizar la fiabilidad de la medición, considerando el método de consistencia interna Alpha de Cronbach, utilizando el software estadístico SPSS v26 y obteniendo un resultado de .91 de fiabilidad (ver tabla 1), que de acuerdo con Carmines y Zeller (1979, citado por Quero, 2010), "Los investigadores consideran, que como regla general, las confiabilidades no deben ser inferiores a 0.80 ".

Tabla 1. Resumen de resultados de análisis de fiabilidad alpha de Cronbach.

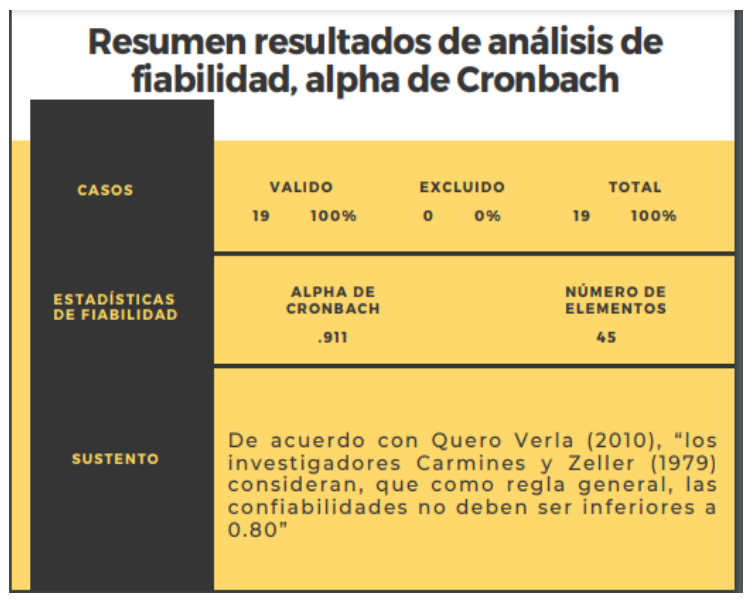

Fuente: Elaboración propia.

El instrumento fue aplicado al objeto de estudio conformado por 19 empresas de la región pertenecientes a la unión de artesanos.

Etapa 3. Análisis estadístico de datos recolectados. Posterior a la aplicación del instrumento de recolección de medición de gestión empresarial, se llevó a cabo la captura y análisis de la información obtenida para su procesamiento en el software estadístico SPSS, en el que mediante el análisis de estadística descriptiva, se identifican las principales problemáticas que refieren a la poca afluencia de clientes y por consecuencia un bajo ingreso por concepto de ventas para los empresarios entrevistados de la unión de artesanos.
Etapa 4. Propuestas para mejorar la funcionalidad de los negocios. Con base en el análisis de resultados y atendiendo a las demandas actuales de la pandemia por COVID-19.

Las 4 etapas previamente señaladas como parte de la metodología de la presente investigación se fundamentan en la metodología propuesta por NODESS, sin embargo, para su implementación como intervención se realizaron acciones de adaptación a las condiciones del contexto propuestas por los autores.

\section{RESULTADOS}

A partir de la metodología aplicada se genera como primer resultado y en acuerdo con los artesanos integrantes de la unión objeto de estudio, que la finalidad de la intervención realizada se cumplió al coadyuvar y promover la creación, organización y fortalecimiento de las empresas del sector artesanal bajo el esquema de la economía social y solidaria en la región Pátzcuaro, Michoacán. Bajo los ejes transversales de sustentabilidad, innovación social, trabajo decente, juventud, género e inclusión, e impulso y desarrollo empresarial, desde la perspectiva teórica de la Economía Social y Solidaria.

En la siguiente figura, se muestra la estructura inicial de intervención del NODESS Pátzcuaro, en la que el principal actor y coordinador es el ITSPA.

Figura 3. Actores principales del NODESS Pátzcuaro-Zirahuén.

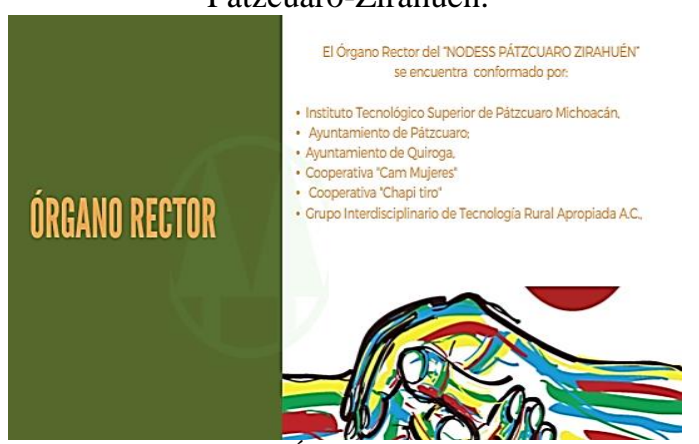

Fuente: Coordinación de Organo rector NODESS PátzcuaroZirahuén.

En un primer momento se realizaron talleres sobre Economía Social y Solidaria, la creación 
del NODESS Pátzcuaro-Zirahuén, su objetivo $\mathrm{y}$ funcionalidad. A partir de las 20 sesiones que constituyeron dichos talleres ofertados a los artesanos, se logró la capacitación y el involucramiento de agentes participantes en el INAES denominada primera formación "NODESS: Dinamizadores de Economía Social y Solidaria".

Con respecto a la aplicación del instrumento de recolección de datos, se obtuvo información importante en aspectos de: ingresos, producción, mercadotecnia, innovación y recursos humanos.

Los empresarios refieren que una de sus principales necesidades de capacitación es en el área de administración, contabilidad, mercadotecnia y uso de Tic's (figura 4). Aunado a ello, con respecto al uso de redes sociales y ventas por internet se identifica que 15 de las 19 empresas no hacen uso de esta herramienta para sus ventas (figura 5). Y se corrobora en los datos obtenidos con respecto al incremento de ventas en los últimos 3 meses, en donde, 16 de las empresas se muestran en desacuerdo (figura 6).

Figura 4. Necesidades de capacitación.

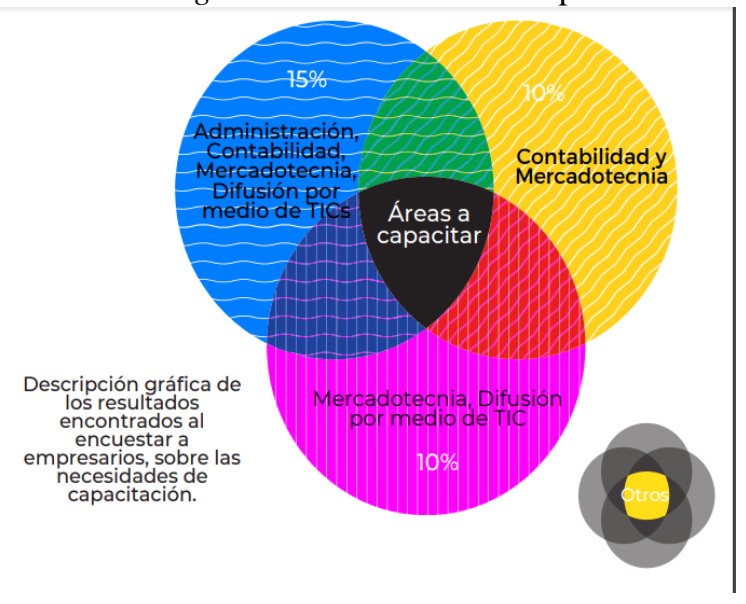

Fuente: Elaboración propia.
Tabla de Frecuencias figura 4

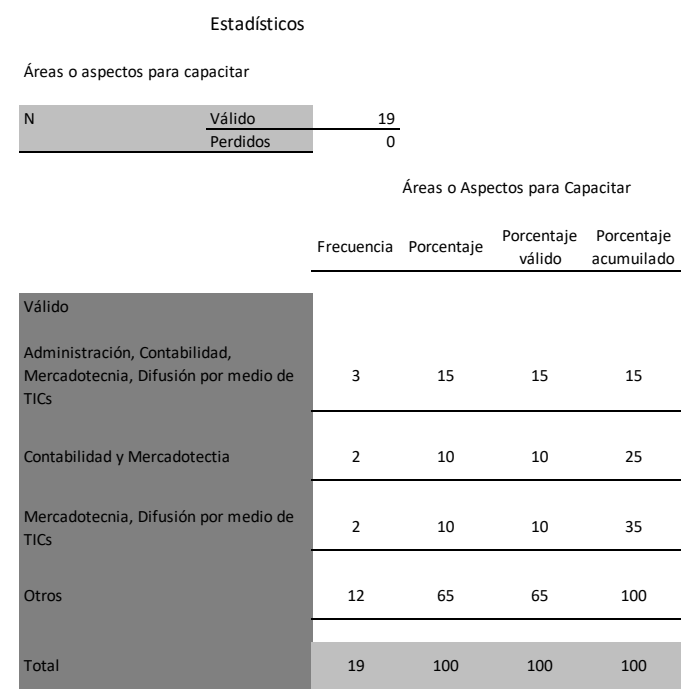

Figura 5. Uso del Internet y Redes Sociales.

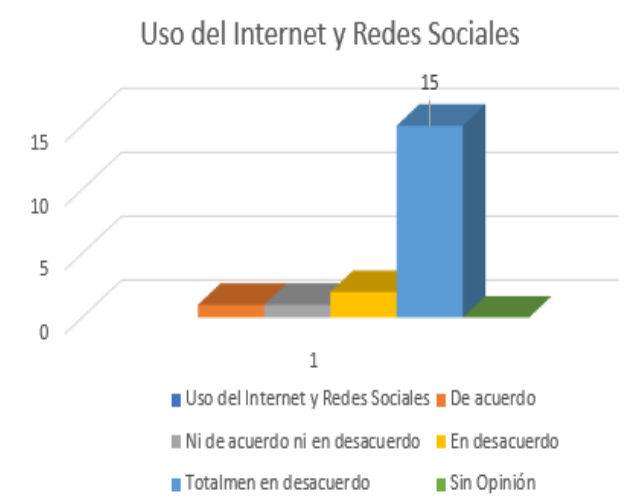

Fuente: Elaboración propia. 
Tabla de Frecuencias figura 5

Frecuencias

Estadísticos

Uso del Internet y Redes Sociales

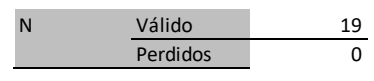

Uso del Internet y Redes Sociales

Frecuencia Porcentaje Porcentaje Porcentaje válido acumuilado

\begin{tabular}{|c|c|c|c|c|}
\hline Válido & & & & \\
\hline De acuerdo & 1 & 5 & 5 & 5 \\
\hline $\begin{array}{l}\mathrm{Ni} \text { de ecuerdo ni en } \\
\text { desacuerdo }\end{array}$ & 1 & 5 & 5 & 5 \\
\hline En desacuerdo & 2 & 11 & 11 & 11 \\
\hline $\begin{array}{l}\text { Totalemente en } \\
\text { desacuerdo }\end{array}$ & 15 & 79 & 79 & 79 \\
\hline Total & 19 & 100 & 100 & 0 \\
\hline
\end{tabular}

Figura 6. Incremento de ventas. Aumento de Ventas

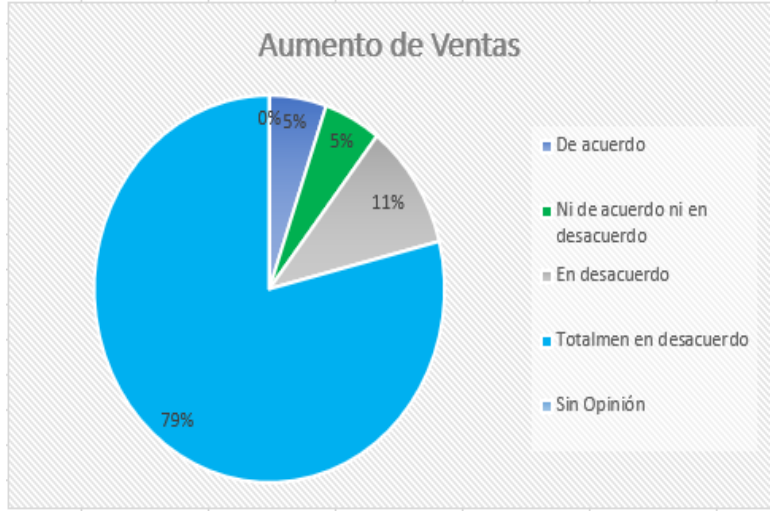

Fuente: Elaboración propia.
Tabla de Frecuencias figura 6

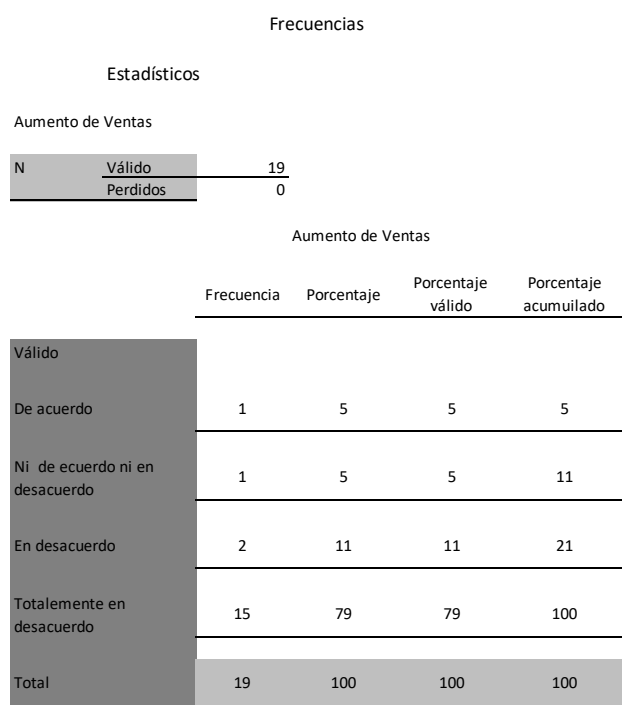

Los resultados mostrados con anterioridad son los de mayor relevancia para el sustento del presente tema de estudio, ya que a partir de ello, se puede identificar como principal área de oportunidad la disminución de los ingresos, el bajo uso de herramientas de las tic's y la falta de la filosofía de la empresa, esta última base del modelo de negocios.

A partir de ello, se consolida una propuesta de mejora y alternativas de solución para contrarrestar su principal problema: bajas ventas e ingresos insuficientes.

Dentro de las propuestas de acciones estratégicas resultado de la intervención se puede resaltar por el impacto de las mismas dos principalmente: la primera fue el diseño organizacional de los negocios para consolidar su modelo operativo de negocios y la segunda corresponde al diseño de una página Web (http://artesaniaspatzcuaro.000webhostapp.co $\mathrm{m} /$ ), dando una alternativa factible para el incremento de las ventas para los artesanos, mediante el comercio electrónico 
Figura 7. Portada de página web como resultado de intervención.

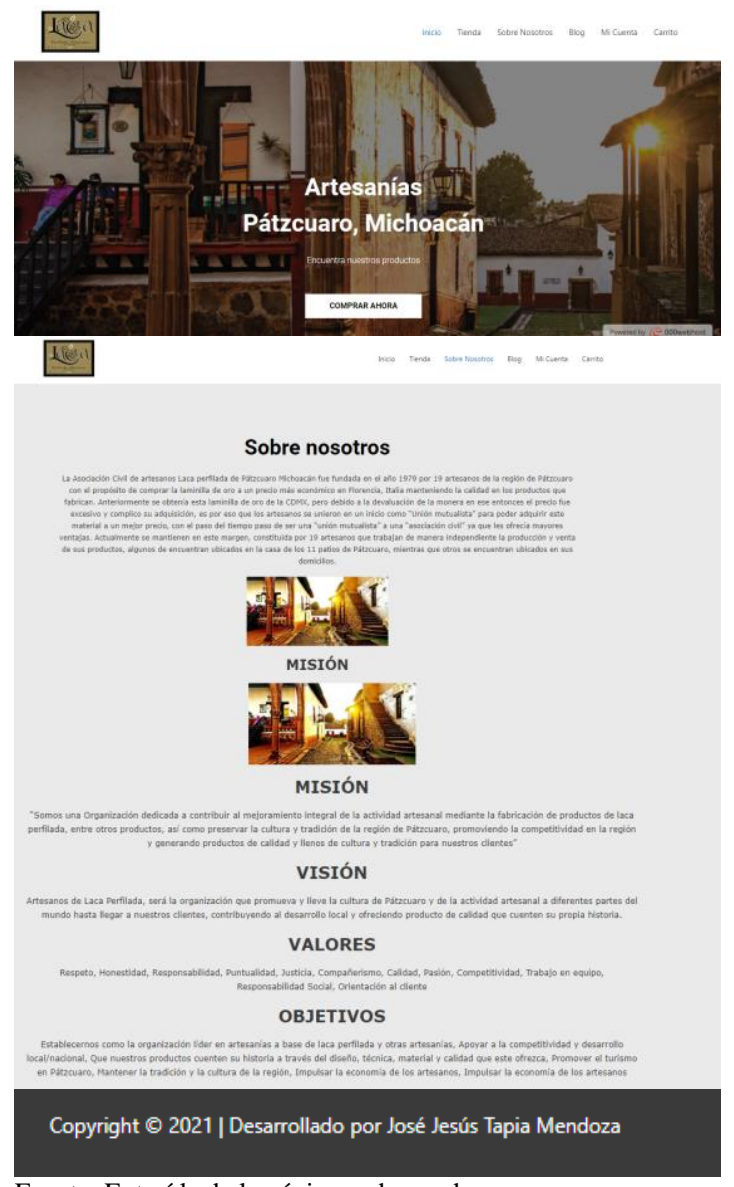

Fuente: Extraída de la página web creada.

Para el diseño y puesta en marcha de las dos estrategias previamente mencionadas se promovió el trabajo colaborativo entre empresarios, investigadores y alumnos en formación integral, mismo que se propone como parte de la conformación de un NODESS.

\section{CONCLUSIONES}

El ITSPA, a través del trabajo colaborativo entre el cuerpo docente, alumnos, sectores sociales y de gobierno, promueve la creación, el fortalecimiento y el desarrollo de los negocios del territorio regional, tal como lo muestran los resultados de esta investigación, ya que como lo afirman Eslava-Zapata, Chacón-Lobo, Chacón-Guerrero, Édixon, \& Gonzalez-Júnior (2018, p. 102) "la relación sector productivo-Universidad se hace hoy prioritaria, pues de allí depende el desarrollo de la capacidad científica y tecnológica de un país. En efecto, la renovación y actualización del recurso humano con habilidades y conocimientos científicos representa la base para intervenir en una sociedad cada día más exigente y con una economía altamente competitiva".

Con esta investigación se aportan conocimientos especializados referentes a las áreas identificadas como necesarias para el incremento de las ventas y el fortalecimiento del modelo de negocios, para con ello, se permita la mejora de la Economía Social y Solidaria de la región, contribuyendo de manera puntual con el eje transversal de creación y mantenimiento del empleo, por lo que el fín planteado de la presente investigación se cumple al realizar una intervención a través de un NODESS como estrategia para las MIPyMES de una unión de artesanos de la región Pátzcuaro Michoacán, ante la pandemia COVID-19.

A partir de la intervención realizada con el enfoque de la metodología de un NODESS se pudo identificar la problemática actual ocasionada por la pandemia de COVID19. Esta contingencia vino a alterar la operatividad del sector salud, y además una alta afectación de otros sectores, principalmente el empresarial, por lo que resulta indispensable aplicar acciones estratégicas que minimicen el impacto de las amenazas del contexto, tales como: el uso de las Tic's como estrategia para incremento de las ventas, capacitación continua y la adopción de una filosofía empresarial como parte de su modelo de negocios. Anzules, et.al., llegaron a conclusiones similares en un estudio sobre el uso del comercio electrónico de las PyMES al norte de Guayacil destacando que la innovación es fundamental para garantizar nuevas formas de comercializar, pues al incrementar los ingresos, se pueden ampliar los métodos y herramientas para lograr mayor rentabilidad.

La participación social y de los diferentes actores es una estrategia prioritaria mundial que puede garantizar una mejora de las condiciones actuales. Un NODESS como estrategia puede incrementar la resiliencia empresarial de las MIPyMES y así tener un impacto positivo en el desarrollo económico 
de la región.

Con este estudio se detectaron las áreas de oportunidad, para generar propuestas de apoyo para el crecimiento de las MIPyMES, encaminadas a mejorar las estrategias de comercialización bajo un enfoque del uso de las Tic's, que atiende las demandas de los clientes ante las circunstancias de la pandemia por COVID-19. 


\section{REFERENCIAS}

Adame, R. L. (2017). El ambiente de negocios y su influencia en el desarrollo empresarial de las micro y pequeñas empresas del sector turístico de Pátzcuaro, Michoacán. (Tesis inédita de Doctorado, Universidad de Durango, Morelia, Michoacán).

Anzules, R.B. Bajaña, R.N. y Mazano, L. (2020) El uso del comercio electrónico y su incidencia en la rentabilidad de las PYMES del norte de Guayaquil. Revista Observatorio de la Economía Latinoamericana. https://www.eumed.net/rev/oel/2020/03/comercio-electronico-pymes.html http://hdl.handle.net/20.500.11763/oel2003comercio-electronico-pymes

De la Cruz May, S.. Y May Guillermo, EG (2021). Prácticas de innovación implementadas por Mipymes en el sector de la restauración ante el COVID-19 en Tabasco, México. Nova Scientia, $13(\mathrm{e})$.

https://doi.org/10.21640/ns.v13ie.2834

Eslava-Zapata, R., Chacón-Lobo, G., Chacón-Guerrero, Édixon, \& Gonzalez-Júnior, H. A. (2018). Cooperación académica: Universidad y sector productivo regional. Revista Perspectivas, $3(1), 102-114$.

https://doi.org/10.22463/25909215.1427

Gil, C. G. (2018). Objetivos de Desarrollo Sostenible (ODS): una revisión crítica. Papeles de relaciones ecosociales y cambio global, 140, 107-118.

Guardado López, S. C., Martínez Flores, J., \& Tapia Torres, D. E. (2020). COVID-19 en México: repercusiones y retos del factor humano, financiero y fiscal. Ciencia, Economía y Negocios, 4(2), 5-32.

https://doi.org/10.22206/ceyn.2020.v4i2.pp5-32

INAES. (2020). Términos de referencia para la formulación de la Propuesta de Trabajo [Archivo PDF].

https://www.gob.mx/inaes/

León, O. \& Palma, E. (2018). Aplicación de las Tecnologías de Información y comunicación en los procesos de innovación empresarial. Revisión de la literatura. I+D Revista de investigaciones, 11(1), 144-152.

https://doi.org/10.33304/revinv.v11n1-2018012

Medina, A.L., Flores, U.(2020). Análisis de la política de desarrollo social en el estado de Oaxaca, México 2013-2016. La economía social como propuesta. Boletín de la Asociación Internacional de Derecho Cooperativo. 56(1), 73-105.

http://dx.doi.org/10.18543/baldc-56-2020pp73-105

Ochoa, G. S., Medina, A.C., Santamaria, B.L.Y., Ramírez, C. D., Paramo, R. E. y Ochoa, N.E. (2020). Reto de las mipymes para incursionar en el marketing digital en tiempos de COVID19. RHS: Revista Humanismo y Sociedad. 8(2), 132-145. https://dialnet.unirioja.es/servlet/articulo?codigo $=7716810$

Quero, V. M. (2010). Confiabilidad y coeficiente Alpha de Cronbach. Telos. 12 (2), 248-252. http://www.redalyc.org/articulo.oa?id=99315569010

Rodríguez, K. G., Ortiz, O. J., Quiroz, A. I., y Parrales, M. L. (2020). El e-commerce y las Mipymes en tiempos de Covid-19. Espacios. 41(42), 100-118.

https://doi.org/10.48082/espacios-a20v41n42p09

Villegas-Garza, A.S. (2020). La transición imperativa del marketing del comercio físico al marketing del comercio online, implementando la experiencia de compra durante la pandemia del COVID-19 implementando la experiencia de compra durante la pandemia del COVID-1. $\begin{array}{lllll}\text { Revista DYCS } & \text { Victoria. } & 2 & \text { (2), } & \text { 59-67. }\end{array}$ https://dycsvictoria.uat.edu.mx/index.php/dycsv/article/view/70/38 